\title{
ONE UNEQUAL ERROR CONTROL METHOD FOR TELEMETRIC DATA TRANSMISSION
}

\author{
Tomáš Hirner ${ }^{*}$ — Peter Farkaš ${ }^{* *}$ — Srečko Krile ${ }^{* * *}$
}

\begin{abstract}
In wireless sensor networks (WSN) it is necessary to use very simple codes for transmission of information since the nodes in these networks have usually only limited energy available not only for transmission but also for processing. On the other hand, common codes do not usually take into account the fact that in case of telemetric information the weights of individual orders are not equal and errors in different orders cause different deviations from correct value. In this contribution, new very simple codes for transmission of telemetric information on WSN will be presented, which take into account the abovementioned requirements. Resulting square deviation will be used as a quality evaluation criterion.
\end{abstract}

K e y w or d s: telemetric information, resulting square deviation (RSD), low-density parity-check (LDPC) codes, unequal error control (UEC), wireless sensor networks (WSN)

\section{INTRODUCTION}

Telemetry is a field dealing with measurement and transmission of so called telemetric information or telemetric data. In telemetric data, the weights of individual orders are not equal and errors in different orders cause different deviations from correct value. Therefore for objective qualitative evaluation of telemetric systems, only the statistical criteria that take into account this fact are appropriate. One of such criteria is resulting square deviation $\delta_{v}^{2}$ [1] usually used in connection with telemetric data transmitted via a discrete channels. This criterion will be used also in this paper for comparison of the new unequal error control method proposed in this paper for its evaluation and comparison with other related methods.

Usually the values of resulting square deviation are obtained via simulation of the telemetric system using computer. Analytical method for calculation of resulting square deviation was proposed in [1] but only for quite restricting round conditions eg for transmission via binary symmetric channel (BSC) without using any error control code. Later the analytical method was introduced for its calculation in [2] which allowed calculate $\mathrm{RDS}$ value also in case that the transmitted telemetric data was protected via linear block code with known weight distribution. Description of one error control coding method adapted on the basis of resulting square deviation for telemetric data transmission was presented in [3]. Low - density parity - check (LDPC) codes (sometimes called Gallager codes) were introduced by Gallager in his PhD. thesis and then published also in [4]. LDPC codes remained largely unstudied for over thirty years, with only scattered references to them appearing in the literature.
After rediscovery of the codes by Mc Kay [5] there was explosion of research and publication activities around LDPC codes because they belong to so called capacity achieving codes, but only few were devoted to unequal error correction with LDPC codes, for example [6]. Today the LDPC codes found their way into standards [7]. Other quite important advantage from the point of view of telemetric information coding is the flexibility of these codes. Almost any rate and block length for LDPC codes can be created simply by specifying the shape of the parity check matrix.

This article presents a new very simple method for error control coding of telemetric data with the purpose to reduce the resulting square deviation caused by the transmission of the data through discrete channel with noise. The new method will be denoted as unequal error control of bits by importance groups and it will be compared with the method of unequal symbol repetition [3]. The paper is organized as follows. First, the criterion RSD will be mentioned briefly in Section 2, then the method will be described in Section 3. Then we will in detail describe method for unequal error control of bits by importance groups in Section 4, later the comparison will be made in Section 5. Some concluding remarks will be given in Section 6 .

\section{RESULTING SQUARE DEVIATION}

Resulting square deviation [1-3] was selected for qualitative evaluation of the transmission errors impact on

\footnotetext{
* Institute of Telecommunications, Slovak University of Technology, Ilkovičova 3, 81219 Bratislava, tomas.hirner@gmail.com, p.farkas@ieee.org, ${ }^{* *}$ Faculty of Informatics, Pan European University, Nevädzová 5, 82101 Bratislava, Slovakia, ${ }^{* * *}$ Department of Electrical Engineering and Computing, University of Dubrovnik, Cira Carica 4, 20000 Dubrovnik, Croatia, srecko.krile@unidu.hr
} 


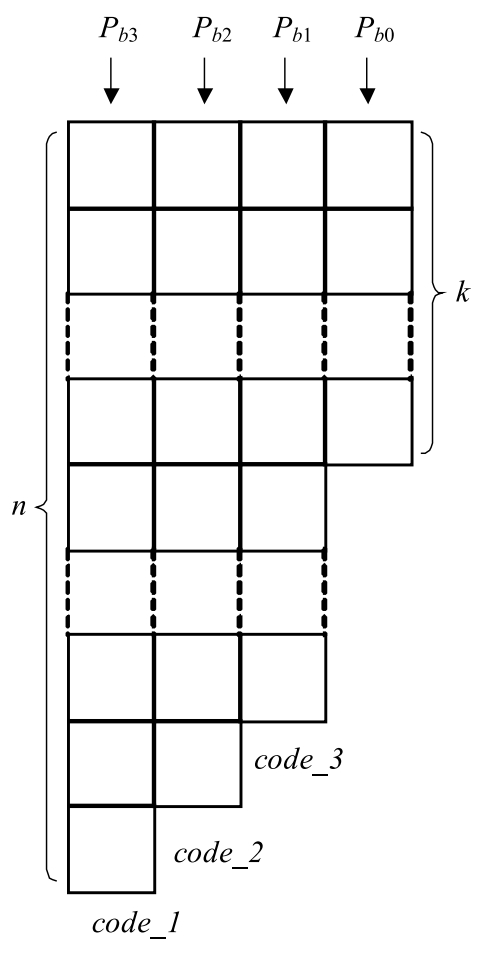

Fig. 1. Method of unequal error control by block codes

telemetric data. It is defined as follows

$$
\delta_{v}^{2}=\frac{1}{\left(2^{k}-1\right)^{2}} \sum_{\alpha=0}^{2^{k}-1} P(\alpha) \sum_{\beta=0}^{2^{k}-1}(\beta-\alpha)^{2} P(\beta / \alpha)
$$

where $P(\alpha)$ is the probability of sending the number $\alpha$ into discrete channel, $P(\beta / \alpha)$ is the probability of receiving number $\beta$ provided that number $\alpha$ was sent and $(\beta-\alpha)^{2}$ is squared error that will result from changing the number $\alpha$ into $\beta$.

\section{METHOD OF UNEQUAL ERROR CONTROL BY BLOCK CODES TRANSMISSION CHANNEL}

In this section, we will briefly describe the basic simple idea for unequal error control coding based on block codes introduced in [8]. It is illustrated in Fig. 1.

The procedure starts with number of identical length telemetry information blocks arrangement below each other. They form first $k$ rows from top in Fig. 1. Redundancy is not assigned as usually to each information part of row code words separately, but instead it is assigned to individual columns. Each column is represented by certain weight. In the example in Fig. 1, the length of information parts of column code words $k$ is four. This arrangement allows using codes with different redundancy (or in other words different error control capability) to different columns with different weights. In most cases, different coding technique will be implemented to each column. Different error control capability of the codes can be characterized also by different bit error rate $P_{b}$ on the output on corresponding decoder if the data are transmitted via BSC. Selection of the codes for different orders depends on the coding method and on the error rate $p$ in BSC. As already mentioned, importance (weights) of each information symbol in the original row blocks of telemetric data vary and errors in different orders result in different deviations from correct value. Now it is quite obvious that the method has to assigned stronger error control codes to higher orders in order that they are protected better than the lower orders. Heuristically it was decided, that the lowest order will not be protected by an error control code to keep the overall redundancy as low as possible. Therefore the resulting bit error rate for this order will be identical to the bit error rate of BSC. In the example depicted in Fig. $1 P_{b 3}$ denotes bit error rate for the highest order. $P_{b 0}$ stands for bit error rate for the lowest order, (zero order). Then the codes have to be selected in accordance with the following relationship: $P_{b 3}<P_{b 2}<P_{b 1}<P_{b 0}$.

Bit error rate for the weight of the lowest order $P_{b 0}$ will be considered the error rate in $\mathrm{BSC}$, ie $P_{b 0}=p$. We do not implement any code in the lowest order, since in comparison with other orders the information in this order has the lowest importance.

For some linear block error control codes it is possible to calculate exact bit error rate $P_{b}$ but to do it the weight spectrum of the code must be known $[2,9]$, (which is not always the case)

\section{$P_{b}=$}

$$
\sum_{j=1}^{n} \frac{j}{n} n_{j} \sum_{v=0}^{t} \sum_{r=0}^{v}\left(\begin{array}{c}
j \\
v-r
\end{array}\right)\left(\begin{array}{c}
n-j \\
r
\end{array}\right) p^{j-v+2 r}(1-p)^{n-j+v-2 r}
$$

where $n_{j}$ is the number of code words with the weight $j$.

Resulting square deviation $\delta_{v}^{2}$ can then be calculated using formula from [2]

$$
\delta_{v}^{2}=\frac{1}{\left(2^{k}-1\right)^{2}} \sum_{j=0}^{k-1} 2^{2 j} P_{b}
$$

Since in our case when we focus on one code word from the whole group of code words, each information bit of the code word has a different value of bit error rate $P_{b}$, we must modify the relation (3) to relations [8]

$$
\delta_{v}^{2}=\frac{1}{\left(2^{k}-1\right)^{2}} \sum_{j=0}^{k-1} 2^{2 j} P_{b_{j}}
$$

The analysis in [8] showed, that the presented method if used with appropriately selected $\mathrm{BCH}$ code to protect the highest order of telemetric information only, achieved better performance in terms of telemetric data deviation than the method with unequal symbol repetition [3]. 


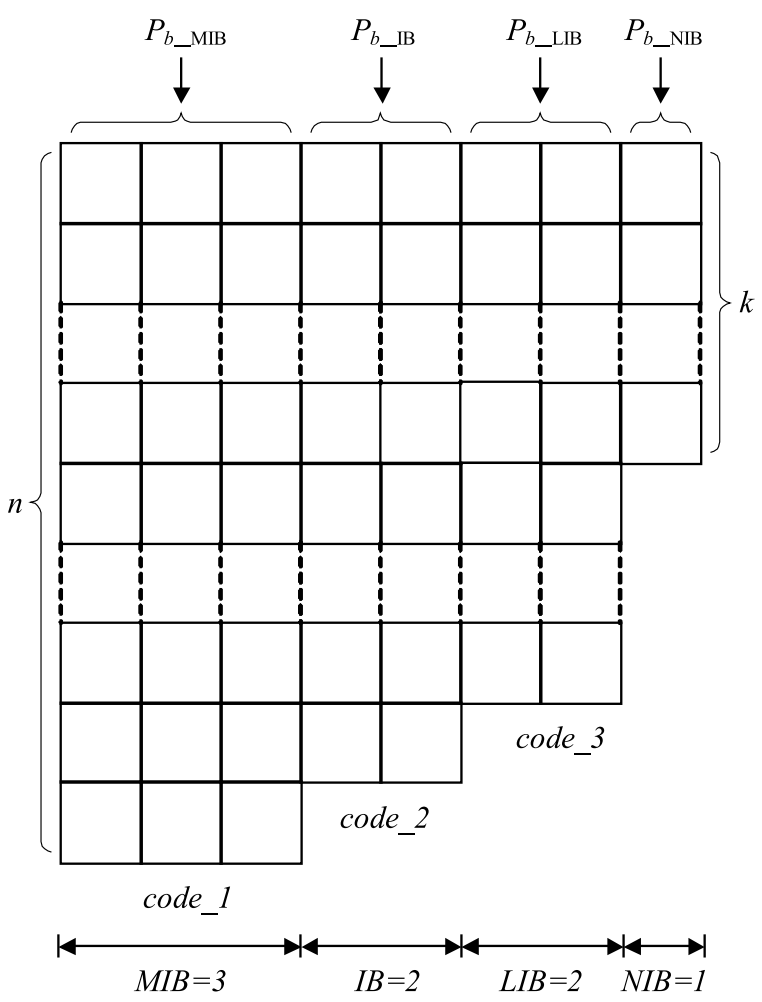

Fig. 2. New proposed method for UECBIG

\section{METHOD FOR UNEQUAL ERROR CONTROL OF BITS BY IMPORTANCE GROUPS DATA LINK LAYER}

In modern systems eg for video or audio transmission also other situation can arise, namely that the importance or weights of groups of symbols are equal.

In new method information bits are divided into four importance groups. Let

- MIB - most important bits,

- IB - important bits,

- LIB - less important bits,

- NIB - no important bits.

The new method will therefore not protect only the highest order of telemetric data as in [8], but also other orders belonging to "important groups. The other main innovation if compared with [8] is that except $\mathrm{BCH}$ codes also LDPC codes will be allowed to protect particular so called importance groups of symbols. This method will be called method for unequal error control of bits by importance groups (UECBIG).

The question can arise what will be the RSD if telemetric data will be transmitted via such scheme.

To answer this question, let us describe and analyze the new method in more detail for an example with row block with lengths 8 illustrated in Fig. 2. Four importance groups are distinguished. The NIB group will not be protected by an error control code as it has the lowest importance. Let the number of columns for particular groups be distributed as follows: $M I B=3, I B=2, L I B=2$ and $N I B=1$. Three different error control codes will be selected in order to achieve unequal error protection of corresponding groups: The resulting bit error rates (on the output of decoders) belonging to corresponding importance groups will be denoted: $P_{b_{-} M I B}, P_{b_{-} I B}, P_{b_{-} L I B}$ and $P_{b_{-} N I B}$. It could be seen that in contrast to method in [8], not every individual bit (in different order) will have different bit error rate, but individual groups of information bits will be characterized by different bit error rate. Bit error rate for the weight of the lowest order $P_{b_{-} N I B}$ will again be considered to be equal to the BSC error probability $P_{b_{-} M I B}=p$.

The following formula presents resulting square deviation $\delta_{v}^{2}$ of the new method for unequal error control of bits by importance groups

$$
\begin{aligned}
\delta_{v}^{2} & =\frac{1}{\left(2^{k}-1\right)}\left[\left(P_{b_{-} N I B} \sum_{j=0}^{N I B-1} 2^{2 j}\right)+\left(P_{b_{-} L I B} \sum_{j=N I B}^{N I B+L I B-1} 2^{2 j}\right)\right. \\
& \left.+\left(P_{b_{-} I B} \sum_{j=N I B+L I B}^{N I B+L I B+I B-1} 2^{2 j}\right)+\left(P_{b_{-} M I B} \sum_{j=N I B+L I B+I B}^{k-1} 2^{2 j}\right)\right]
\end{aligned}
$$

Analytical computation using (5) is slightly simpler than using (4) because it is not necessary to calculate the resulting bit error rate for each order separately. Relation (5) is general and applies to optional information words length.

\section{COMPARISON OF RESULTS APPROACH TO IMPLEMENTING OF CODING AT DATA LINK LAYER}

In this section we compare the new proposed method UECBIG with the known method proposed in [3]. For these codes analytical methods could be used for calculation of $\delta_{v}^{2}$. To make the comparison as fair as possible it is necessary to chose similar Code rates of compared codes. Code rate $R_{k}$ is defined as number of information symbols $k$ in a code word from the code divided by the number of all symbols $n$ in a code word

$$
R_{k}=\frac{k}{n}
$$

For the new method proposed in this paper analytical approaches for evaluation of $\delta_{v}^{2}$ are not known, therefore computer simulations were used for it. Following codes were selected for comparison:

Code 1 (C1) is regular LDPC code that has variable node degree 5 and check node degree 6 with $n=210, k=$ 35 from [11]. The degree distribution of this code is given by $\lambda(x)=x^{4}$ and $\rho(x)=x^{5}$, an MB algorithm (Majority Based) of order zero $\left(M B^{0}\right)$ has a better threshold than that of the GA [11].

Code 2 (C2) is irregular LDPC code with $n=200$, $k=100$ from [10]. The degree distributions for this code, 


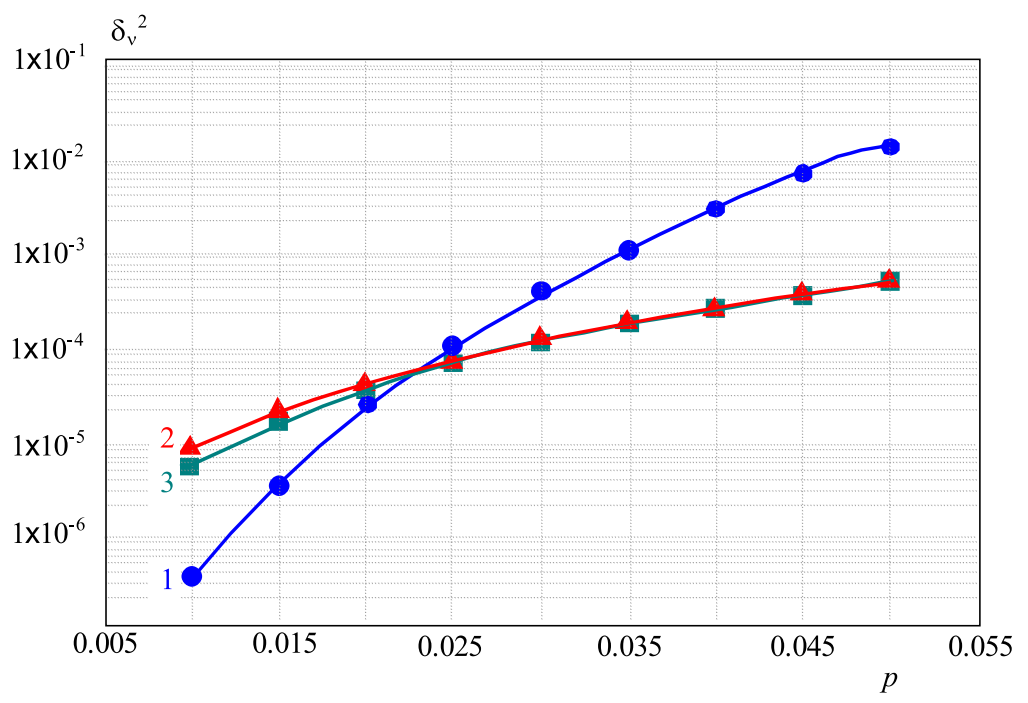

Fig. 3. Dependence of $\delta_{v}^{2}$ on $p$ for following encoding schemes: 1 - new method with $R_{k} \cong 02932$. (LDPC codes used for MIB and IB, $\mathrm{BCH}$ code for LIB and no coding for NIB); 2 - repetition code C5 with $R_{k} \cong 0.3333$ ([5,5,5,3,3,1,1,1]); 3 - repetition code C4 with $R_{k} \cong 02857([5,5,5,3,3,3,3,1])$.

which were optimized for the BSC and GA (Gallager algorithm A) are given by $\lambda(x)=0.1115 x^{2}+0.8885 x^{3}$ and $\rho(x)=0.26 x^{6}+0.74 x^{7}$.

Code 3 (C3) is a BCH code with $n=15, k=7$.

Code 4 (C4) is an repetition code with $n=28, k=8$ unequal number of repetitions for some orders (number of repetitions in $i$-th order is denoted as $r_{i}: r_{7}=r_{6}=r_{5}$, $r_{4}=r_{3}=r_{2}=r_{1}=3, r_{0}=1$ or in shorter notation: $[5,5,5,3,3,3,3,1] . R_{k}=\frac{2}{7} \cong 0.2857$.

Code 5 (C5) is an repetition code with $n=24, k=8$ unequal number of repetitions given by following short notation: $[5,5,5,3,3,1,1,1] . R_{k}=\frac{1}{3} \cong 0.3333$.

Bit error rate values $P_{b}$ for $\mathrm{BCH}$ code could be calculated using $(2)$. Bit error rate values $P_{b}$ for $(200,100)$ irregular LDPC code and $(210,35)$ regular LDPC code are from simulation described in [10-12]. In Fig. 1 there are results of comparison of the new proposed coding method, which were used to encode 700 telemetric messages with 8 orders each. The new coding method was represented by the following scheme: in orders 7, 6 and $5 \mathrm{C} 1$ was used, for orders 4, $3 \mathrm{C} 2$, for orders 2, $1 \mathrm{C} 3$ and for order zero no coding technique is used. For encoding of the 700 bytes of the telemetric information altogether 20 code words from C1, 7 code words from C2 and 100 code words from $\mathrm{C} 3$ were needed. The overall code rate than was: $R_{k}=\frac{5600}{19100} \cong 0.2932$, which is between the values of code rates of C5 and C6.

From Fig. 3 we can see that the new method outperforms both repetition codes for $p \leq 0.025$. In practical applications the channels are in most cases characterized by error probabilities which are much smaller than 0.025 . On the other hand the slight disadvantage of the new scheme is that much longer code words are used for encoding and obviously much more complicated decoding schemes are necessary for decoding of the LDPC codes than for repetition codes.

For repetition codes simple majority decoding could be used, but in order to exploit appropriately the excellent error correcting properties of LDPC codes it is necessary to use a Sum-product or at least Min- sum algorithms.

\section{CONCLUSION}

In this manuscript new scheme for error control coding was proposed suitable for transmission of telemetric data via channels which could be modeled as binary symmetric channels was presented. It is based on using different LDPC codes for different groups of orders in the telemetric data so called importance groups. It was shown by an example in which the new method was compared with repetition codes with unequal number of repetitions that it has an excellent performance for realistic error probabilities (smaller than 0.025). On the other hand the unequal repetition codes are much simpler than the new method and therefore still could be attractive for some applications. Further research on the evaluation of the computational complexity or even better on energetic demands of these schemes could be of interest for such applications as WSN.

\section{Acknowledgement}

This work was supported by Scientific Grant Agency of Ministry of Education of Slovak Republic and Slovak Academy of Sciences under contract VEGA 1/0376/09, 2009-2012. 


\section{REFERENCES}

[1] SMIRNOV, A. S.: Mean Resulting Error at Telemetric Signal Transfer by Residual-less Binary Code (Srednaja rezulturirujushcaja pogreshnosth pri peredache signalov teleizmerenja bezizbytonym dvojinym kodom), Avtomatika i telemekhanika No. 5 (1981), 161-164. (in Russian)

[2] FARKAŠ, P.: Calculation of Resulting Square Deviation Arising During Transmission of Telemetric Data Using Block Linear Self-Repairing Codes, Electronics magazine 39 No. 2 (1988), 154-162.

[3] FARKAS̆, P.—SOTSKOV, J. V.: One of Methods of Telemetric Data Coding, Electronics magazine 38 No. 12 (1987), 897-907.

[4] GallageR, R. G.: Low-Density Parity-Check Codes, MA: MIT Press, Cambridge, 1963.

[5] MACKAY, D. J. C.: Information Theory, Interference and Learning Algorithm, Cambridge University Press, 2003.

[6] RAHNAVARD, N.-PISHRO-NIK, H.-FEKRI, F.: Unequal Error Protection using Partially Regular LDPC Codes, IEEE Trans. Commun. 55 No. 3 (Mar 2007), 387-391.

[7] IEEE (802.16d) Standard 2004.

[8] HIRNER, T.-FARKAS̆, P.: Method for Unequal Error Control by Block Codes for Transmission of Telemetric Information in Wireless Sensor Networks, In: RTT 2006. Research in Telecommunication Technology: 7th International Conference. Nové Město na Moravě, Czech Republic, Brno University of Technology, 2006, pp. 220-225.

[9] CLARK, G. C.-CAIN, J. B. : ErrorCorrecting Coding for Digital Communications, Plenum Press, New York, 1982, pp. 18-23.

[10] BAZZI, L.-RICHARDSON, T. J.-URBANKE, R. L. : Exact Threshold and Optimal Codes for the Binary-Symmetric Channel and Gallagers Decoding Algorithm A, IEEE Trans. Inf. Theory 50 No. 9 (Sep 2004), 2010-2021.

[11] Threshold Values and Convergence Properties of Majority-Based Algorithm for Decoding Regular Low-Density Parity-Check Codes, IEEE Trans. Commun. 52 No. 12 (Dec 2004), 2087-2097.

[12] XIAO, H.-BANIHASHEMI, A. H.: Estimation of Bit and Frame Error Rates of Finite-Length Low-Density Parity-Check Codes on Binary Symmetric Channels, IEEE Trans. Commun. 55 No. 12 (Dec 2007), 2234-2239.

Received 23 March 2010

Tom Hirner was born in 1983 in Bratislava, Slovakia. He received his Bc 2006 and Ing (MSc) 2008 in Telecommunications from Slovak University of Technology (STU) in Bratislava. He is currently with Slovak Telecom, a. s. from
2007. He is now also external PhD student at Institute of Telecommunications, (STU). His research interests are concentrated on Unequal error control coding for telemetric information.

Peter Farkaš (Prof, Ing, DrSc), (MIEEE, URSI) received the Ing (MSc) degree from (STU) in 1980, PhD degree from St. Petersburg State Polytechnic University in 1987 and DrSc degree from STU in 1997. He is with Institute of Telecommunications at STU, since 1999 as a Professor. His research interests include Error Control Coding, Communications Theory, MC CDMA, Wireless Sensor Networks and Software Defined Radio. From 2002 till 2007 he was Visiting Professor at Kingston University, UK and senior researcher at SIEMENS PSE. From SIEMENS he received two awards. In 2003 SIEMENS named him VIP for his innovations and patents. In 2004 he was awarded with the Werner von Siemens Excellence Award for research results on two-dimensional Complete Complementary Codes. He was responsible leader of a team from STU in NEXWAY (Network of Excellence in Wireless Applications and technology) FP5 IST 2001-37944 and also project leader on behalf of STU in CRUISE (Creating Ubiquitous Intelligent Sensing Environments) FP6 IST-2005- 4-027738, (2006-2007). (Projects funded by the European Community).

Srečko Krile was born in 1957 in Dubrovnik, Croatia. Diploma thesis 1980; Master thesis 1988; Doctoral thesis 2000 all at University of Zagreb, Faculty of Electrical Engineering and Computing. From 1983 to 1991 he worked on planning and maintenance of functional communication networks for government. Since 1991 he is with University of Dubrovnik, former Polytechnic and Maritime Faculty. He was the first chief of the Electrical Engineering and Computing Department, currently at a position of Associate Professor. Beside two books: "Electronic Communications in Shipping", part I, and part II: "Mobile Satellite Networks" he also published many scientific papers and has presented them on many international conferences. Research areas: QoS, resource management and optimal capacity allocation, traffic engineering, mobile satellite networks. He is member of editorial board for scientific journal "Naše more" from 1993. He was member of management board for Croatian Academic Research Network (CARNet) from 2004 - 2008. He also is member of Croatian union of musicians (HGU) and Croatian society of composers (HDS). 